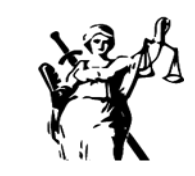

JUSTICIA

ISSN impreso 0124-7441
ISSN digital 2590-4566

\title{
Convivencia escolar en Colombia: una visión jurisprudencial, legal y doctrinal
}

\author{
School coexistence in Colombia: a jurisprudential, legal and \\ doctrinal view
}

\author{
(iD) \\ Olga Sofía Morcote González \\ Universidad de Boyacá., Colombia \\ olgmorcote@uniboyaca.edu.co
}

\author{
Claudia Patricia Guerrero Arroyave \\ Universidad de Boyacá., Colombia \\ pguerrero@uniboyaca.edu.co
}

Recibido: 14 de mayo de 2020 / Aceptado: 26 de julio de 2020 https://doi.org/10.17081/just.25.38.4422

\begin{abstract}
Resumen
En el presente artículo de reflexión se realiza un análisis de la violencia escolar como fenómeno social; a partir de la normatividad de la Convivencia Escolar, la doctrina y el análisis de la jurisprudencial de la Corte Constitucional en la que se enfatiza la protección de los Derechos Fundamentales de los estudiantes por parte de las Instituciones Educativas. Como resultados se encontró que la Corte Constitucional ha creado indirectamente política pública frente al tema direccionando el sistema educativo para que sea un eje promotor de derechos fundamentales. Este trabajo se desarrolló desde la línea de investigación: Desarrollo Humano, Jurídico y Social con un enfoque cualitativo.
\end{abstract}

Palabras clave: convivencia escolar, derechos fundamentales, instituciones educativas, sentencias de la corte constitucional, violencia escolar.

\begin{abstract}
In the present article of reflection an analysis of school violence as a social phenomenon is made; based on the rules of School Coexistence, the doctrine and the analysis of the jurisprudence of the Constitutional Court which emphasizes the protection of the Fundamental Rights of students by the Educational Institutions. This work was developed from the research line: Human, Legal and Social Development with a qualitative approach.
\end{abstract}

KEYWORDS: educational institutions, fundamental Rights, Sentences of the Constitutional Court, School Violence, School Coexistence.

\section{Como Citar:}

Morcote González, O. S., \& Guerrero Arroyave, C. P. (2020). Convivencia escolar en Colombia: Una visión jurisprudencial, legal y doctrinal. Justicia, 25(38), 95-112. https://doi.org/10.17081/just.25.38.4422 


\section{Introducción}

La violencia escolar se ha convirtiendo en las últimas décadas en una problemática de análisis, reflexión e intervención internacional y nacional; en las instituciones educativas fundamentalmente porque son ellas espacios de socialización, convivencia y enseñanza expuestos en algunas ocasiones a situaciones de conflictividad. En la mayoría de casos, la solución que se ha dado a los comportamientos generadores de violencia en los centros de enseñanza ha sido de manera apropiada utilizando los mecanismos alternativos de resolución de conflictos como la conciliación, la mediación, la transacción o la amigable composición; sin embargo; en otras ocasiones la solución no es la correcta, desencadenando en conductas violatorias de los derechos fundamentales de los estudiantes, padres de familia o docentes por parte de los planteles educativos.

Éstas relaciones que se presentan entre niños; adolescentes; docentes; administrativos; directivos y padres de familia; han hecho necesario y visible la realización de estudios e investigaciones relacionadas con la convivencia escolar a nivel internacional y nacional; por estas razones se hace preciso elaborar un análisis reflexivo que permita conocer la historia del fenómeno social, partiendo de las políticas públicas que se han generado para mitigarla, la ley, la doctrina y la jurisprudencia de la Corte Constitucional que han aportado jurídicamente a la solución de esta problemática.

Como antecedente se menciona que la violencia se ha presentado como una constante en la historia de la convivencia escolar; sus primeros estudios se realizaron en la década de los 70 con las investigaciones del psicólogo Dan Olweus, en Noruega, atendiendo al aumento de casos que se presentaron en ese país; es así como el autor, acuña el término "bullying" para referirse a la violencia escolar. Bullying viene del vocablo inglés "bull" que significa toro. En este sentido bullying es la actitud de actuar como un toro en el sentido de pasar por sobre otro u otros sin contemplaciones" (Olweus D. , 1978).

De otra parte, se menciona que para que exista violencia escolar es necesaria la concurrencia de dos partes, las cuales son: "abusador(es) - abusado (s) o víctima (s) -victimario (s), presentándose una conducta negativa, intencional y sistemática de una parte para agredir, ofender, deshumanizar, denigrar o causar daño a la parte pasiva. La relación entre abusador-abusado se define como el comportamiento negativo, repetitivo e intencional (desagradable o hiriente, de una o más personas, dirigido contra una persona que tiene dificultades en defenderse". (Olweus D. , 2004). Según Castillo (2011, p.15), debido a la incidencia de la violencia escolar en la sociedad, a través del tiempo se han realizado investigaciones, apareciendo los primeros informes relacionados con el tema en 1983, en los que Dan Olweus y Erling Roland, realizados en Noruega desde 1973 y luego, se extendieron a los países escandinavos para su estudio. Posteriormente, a finales de los ochenta y principios de los noventa, el fenómeno del acoso entre iguales atrajo cierta atención pública de la investigación en otros países, como Japón, Inglaterra, Países Bajos, Canadá, Estados Unidos y Australia.

Existen teorías que han estudiado el tema; iniciando por el análisis del comportamiento agresivo de los estudiantes, llamada teoría ecológica, su inicio se dio en 1987 fundamentando que las conductas violentas o agresivas que se presentan en los estudiantes, son derivadas del entorno y los diferentes contextos a los que se encuentran expuestos. Esta teoría también expone las dificultades y consecuencias futuras que a partir de la violencia escolar van a sufrir los afectados; al respecto, las investigadoras Carolina Lisboa y Silvia Koller (2009) muestran cómo a diferencia de los años 70 a 90; "hoy es casi consenso en la literatura científica que este proceso de la violencia escolar (acoso) está asociado a graves factores de riesgo en el futuro de las víctimas (suicidio, depresión) como también para los agresores (dificultades de relación con los demás, delincuencia, violencia, entre otros) y exige intervenciones urgentes y puntuales. 
Para acercarnos al tema, se debe partir de un punto de referencia para su desarrollo, siendo de importancia establecer el significado y connotación histórica de la violencia en la humanidad; este término, presente desde el inicio de los tiempos, señala que este flagelo tiene una antigüedad como la que tiene la creación del mundo; cosmogonías, mitologías y leyendas la muestran vinculada a sus orígenes. (Domenach, 1981).

En el presente artículo de reflexión se realiza un análisis con base en la violencia escolar como fenómeno social; a partir de la normatividad de la Convivencia Escolar, la doctrina y el análisis de la jurisprudencia de la Corte Constitucional en la que se enfatiza la protección de Derechos Fundamentales de los estudiantes por parte de las Instituciones Educativa.

Este trabajo se desarrolló desde la línea de investigación: Desarrollo Humano, Jurídico y Social con un enfoque cualitativo. En la primera parte se presentará un enfoque crítico social de la violencia escolar, en la segunda parte se presentará un estado del arte sobre las investigaciones relevantes realizadas en Colombia, en la tercera parte el desarrollo legal y doctrinal, en la cuarta parte el análisis de la jurisprudencia de la Corte Constitucional y finalmente se presentarán las conclusiones.

\section{Método}

El desarrollo del presente artículo se orientará bajo un enfoque cualitativo, deductivo, en el cual se hará una aproximación a los antecedentes de la violencia escolar; posteriormente se revisarán aspectos jurisprudenciales con el ánimo resaltar los pronunciamientos de las altas Cortes en Colombia y las disposiciones orientadas al proceso educativo, finalmente se esbozarán algunas conclusiones que sirvan de soporte para la comunidad académica en general.

\section{Enfoque crítico social de la violencia escolar}

La violencia es un concepto interdisciplinario que es visto por las ciencias sociales como un fenómeno social que se refiere al conjunto de acciones humanas destinadas a ocasionar daño, menoscabo o perjuicio, físico, sexual, económico o psicológico, a una o más personas.

Conforme la Organización Mundial de la Salud, OMS, se ha definido la violencia como una conducta contra uno mismo, contra otra persona, un grupo o una comunidad a través de la utilización de la fuerza física, que tiene como consecuencia o es muy probable que tenga un traumatismo, daño psicológico, problema de desarrollo o la muerte. Éste organismo desde 1996 viene reconociendo la violencia como un problema de salud pública, evidenciando la necesidad de caracterizar las diferentes maneras en que se presenta y los vínculos entre ellas; este organismo internacional dividió la violencia en tres grandes categorías generales a partir de quién comete el acto: violencia autoinfligida que involucra el comportamiento suicida y las autolesiones; violencia interpersonal que ocurre en la familia, la pareja y la comunidad; y la violencia colectiva que se evidencia en ambientes sociales, políticos y económicos. (OMS, 2003).

El sicólogo Zimbardo (2002, p.2), define la violencia como una forma de obrar, que ocasiona daños, maltrato, humillaciones y destruye a personas inocentes; o en hacer uso de la propia autoridad y del poder sistémico, para alentar o permitir que otros obren así en nuestro nombre; para el pedagogo y psicólogo Bandura (2010, p.6), la violencia es una forma de actuar de los seres humanos que se aprende por interrelacion social y su característica principal es que se transmite a través de la observación y el refuerzo; por 
tal motivo es evidente que la violencia se manifieste en las relaciones del hombre a través del tiempo, y aunque se ha exteriorizado de diferentes maneras, ha perdurado en la historia como herencia y parte del cerebro reptiliano1.

Uno de los espacios en los que se ha manifestado la violencia en la humanidad ha sido en el escenario escolar, el cual se origina y desarrolla en las instituciones educativas; espacio de interacción entre niños, adolescentes y adultos; el cual tiene como fundamento la formación y educación de los estudiantes para su futuro desenvolvimiento en la sociedad, es así como, el ámbito escolar se entiende como una institucionalización de aquellos espacios de formación ciudadana y aprendizaje, con el concepto esperanzador de que a partir de el, se van a formar seres integrales, capaces de convivir en sociedad y aportar a la misma para el progreso particular y general de las naciones; por consiguiente es de gran importancia la calidad de las relaciones interpersonales que se presentan en el ámbito escolar y su estudio.

A nivel nacional (Colombia) se han desarrollado investigaciones de violencia y acoso escolar; la mayoría de ellas avanzaron a partir de los años 90 con la Constitución Política de 1991; estableciendo que Colombia es un Estado Social de Derecho, y por lo tanto el Estado debe ser garante constitucional de la efectividad de los principios y los derechos fundamentales de los ciudadanos, lo que conllevó a que los teóricos empezaran a analizar los aciertos y desaciertos del Estado en el cumplimiento de sus fines.

A pesar que con anterioridad a los años 90 la violencia escolar no se había investigado con profundidad; se relaciona su existencia con el contexto social que se venía presentando en esa época con el narcotráfico y terrorismo; y coincide con el entorno violento que estaba teniendo influencia en los comportamientos violentos o agresivos que se presentaban en las aulas de clase, al igual que con los modelos pedagógicos existentes en la formación.

En Colombia se ha confirmado que existen jóvenes que asumen el manejo de la escuela como un lugar para demostrar su fuerza, utilizando la violencia y la intimidación con amenazas a sus compañeros, buscando el lado débil de los maestros, cargando un acumulado de experiencias que les permiten controlar situaciones y personas, es allí donde se verifica la teoría ecológica de la violencia escolar (Medina Gallego, 1991), p.5).

En el Instituto para la Investigación Educativa y el Desarrollo Pedagógico (IDEP), se realizó una investigación sobre el tema en 1999, concluyéndose que "la violencia es una realidad presente en la cotidianidad y se manifiesta desde el simple desconocimiento por el otro cuando no se responde a su pregunta, cuando no se reconoce su cuerpo en el espacio, cuando su nombre nunca es pronunciado, hasta el golpe de autoridad, el uso del conocimiento y la edad para someter, la pretensión de subyugar a los aparentemente más débiles a una voluntad ajena a sus deseos, o el disparo que elimina el contrario, hay un enorme y desafortunado diapasón de acciones que son nuestra cotidianidad". Indica además que: "no se trata, pues, de decir que la escuela es un manicomio, ni una cárcel, ni un cuartel, pero con poco esfuerzo se podría pensar que tiene un poquito de cada cosa2.

El psicólogo e investigador Colombiano Enrique Chaux en el año 2002 se interesó por complementar los estudios e investigaciones elaborados con anterioridad en relación con la violencia escolar en el país, este autor es de gran importancia en el avance investigativo nacional, sus trabajos tienen la característica de mostrar una perspectiva diferente con respecto a la violencia con el propósito de establecer la percepción

1 Mayor información en https://psicologiaymente.com/neurociencias/modelo-3-cerebros-reptiliano-limbico-neocortex

2 Instituto para la Investigación Educativa y el Desarrollo Pedagógico, IDEP. Violencia en la escuela. Alcaldía Mayor de Santafé de Bogotá. 1999. 
directa que tienen los actores de la violencia escolar; narrando en sus entrevistas los asuntos que motivaron la violencia y las estrategias que usaron para manejarlas; las emociones que sentían durante las diferentes etapas de los conflictos, lo que sucedía con las relaciones después de estos, y el rol que jugaban los(as) terceros(as)(Chaux, 2002, p.2).

Chaux, manifiesta en sus estudios que el entorno en que se desarrollan los jóvenes debe analizarse para comprender el origen de las conductas violentas; considerando que si se limita a encontrar la causa y origen de este fenómeno en el entorno escolar, será muy difícil obtener una explicación para ello, haciendo una aproximación al estudio de la agresión en niños y niñas, la cual ha sido buscar las causas de esa agresión y concentrar todos los esfuerzos de intervención en cambiar esas causas, manifestando que el centro escolar, es el espacio o contexto donde se manifiestan los síntomas de una realidad social contradictoria. (Chaux, 2003, p.5).

Otro estudio relevante en el país es el realizado en la ciudad de Barranquilla con 80 niños de edades entre 9,11 y 13 años para describir el fenómeno de la violencia escolar entre ellos y atendiendo a que los mismos tienen diferentes situaciones socioeconómicas, denominado: "Representación sobre el maltrato entre iguales en niñas y niños escolarizados de 9,11 y 13 años de nivel socioeconómico alto y bajo en la ciudad de Barranquilla, Colombia". (Hoyos, Aparicio, \& Heilbron, 2004)

Investigaciones como la mencionada, refleja cómo la violencia escolar con el transcurso del tiempo se ha intensificado; es así como el Ministerio de Educación Nacional viene formulando políticas de convivencia escolar con el fin de atenuar este problema. Estas políticas, buscan la educación integral de los estudiantes; por esto es necesaria la incursión y el compromiso de las diferentes esferas sociales como son la familia, el gobierno, las instituciones y la comunidad, propiciando la formación ciudadana en temáticas como: los mecanismos alternativos de resolución de conflictos.

Sin embargo, debe reiterarse, "es la escuela, la institución que imparte educación, que forma, que construye, y que conforme a lo establecido por la ley 115 de 1994 en su artículo 1, es instrumento a través del cual se presta el servicios de educación, buscando un proceso permanente de formación cultural, social y personal, que tiene como fundamento una concepción integral de la persona humana de su dignidad, de sus derechos y deberes". (Rodríguez, 2011)

\section{Una aproximación al Estado del arte sobre violencia escolar}

Las investigaciones sobre violencia escolar, en su mayoría desde enfoques psicológicos, vienen estudiado el origen de los comportamientos agresivos o violentos del ser humano, y específicamente de los estudiantes que se desarrollan en el contexto educativo; para Blaya, Debarbieux, Rey y Alamillo (2005) los estudios psicoeducativos han focalizado su interés en los procesos personales e interpersonales implícitos y explícitos en el complejo fenómeno de la agresividad, la conducta agresiva, la victimización y la conducta violenta, en sus diferentes matices.

Otra investigación de violencia escolar realizada en Santiago de Chile, desde el enfoque ecológico3 manifestó la manera de estudiar los modelos tradicionales para abordar la agresividad entre pares (como elemento de la violencia en las escuelas) y cómo estos se han enfocado ya sea en los agresores o en las víc-

3 El modelo ecológico considera distintos aspectos que concurren en un hecho generador de violencia como el social, comunitario, relacional e individual. 
timas de la violencia. De esta manera se ha tendido a la búsqueda de perfiles individuales de los agresores y víctimas, buscando establecer los factores causantes de dichos perfiles (Berger, 2009). Son varios los autores que se inclinan por este enfoque para explicar el origen de las conductas agresivas en la escuela, entre ellos también se encuentran:

La organización mundial de la Salud desde el año 2003, esquematizó el enfoque de trabajo sobre la violencia basado en el modelo ecológico, debiendo aplicarlo sobre las diferentes situaciones de violencia como factores que relacionan al individuo con ese comportamiento social, comunitario, relacional e individual; en el nivel individual se consideran importantes los factores biológicos, psíquicos y educativos; en el nivel relacional se encuentran factores relacionados con la familia, pareja y amigos; en el nivel comunitario son importantes la escuela, el barrio y el lugar de trabajo y en el nivel social se encuentran las organizaciones que propagan la violencia y la aceptan (OMS, Informe mundial sobre la violencia y la salud, 2003).

Lo anterior, analiza como el modelo ecológico que estudia la violencia hace una interacción entre los diversos factores que concurren en el ser humano para que su comportamiento sea de cierta manera y no de otra: la investigación realizada en la Universidade do Vale Rio dos Sinos, de la misma manera enfatiza en la prioridad por hacer un debate y aproximación de la violencia en la escuela, su dinámica y consecuencias desde este aspecto. (Abramovay, 2005, p.11).

Existe otra línea de investigaciones relacionada con la violencia escolar; desde un enfoque criminológico, buscando establecer que la violencia escolar, es originada por psicopatologías, lo cual indica que algunos de los actores de la violencia escolar tienden a tener comportamientos a partir de patologías que van a generar perfiles de criminalidad. Al respecto Yunes y Zubarew $(1993$, p.3) especifican que entre las causas de la violencia, las "características individuales, experiencias familiares, relaciones de pares, acceso a armas, consumo de alcohol y otras drogas, exposición a violencia en los medios de comunicación y otros factores políticos, culturales y sociales se interrelacionan y juegan un papel fundamental en la ocurrencia de la violencia en jóvenes" (Tamara-Yunes, 1993) .

Y finalmente se encuentra el enfoque fenomenológico, según el cual la violencia escolar, es un producto social, que nace a partir de las relaciones sociales del hombre, siendo dinámico, cambiante y debe analizarse específicamente como un aspecto social subjetivo, debido a las circunstancias y acontecimientos que se presentan en la sociedad, tienen matices y características específicas que deben estudiarse de manera particular; los autores que se inclinan por el estudio de la violencia escolar desde ese enfoque afirman que ésta, nace a partir del actuar del ser humano.

De otra parte este tema ha sido caracterizado como un fenómeno humano definido como una acción, con una lógica, sin embargo, ésta no se refiere a la coherencia deductiva posible del pensamiento consigo mismo, sino de determinadas lógicas de acción sobre las cuales se instalan las decisiones humanas (Florez González, 2009, p.2).

Durante los años 2005- 2009 la Organización de las Naciones Unidas para la Educación, la Ciencia y la Cultura (Unesco), desarrolló una investigación sobre Violencia escolar, encontrando como resultado que América Latina es la región del mundo donde más violencia escolar se presenta; los resultados del estudio que se realizó en 2.969 escuelas; 3.903 salas de clases y 91.223 estudiantes de sexto grado de 16 países latinoamericanos: Argentina, Brasil, Colombia, Costa Rica, Cuba, Chile, Ecuador, El Salvador, Guatemala, México ,Nicaragua, Panamá, Paraguay, Perú, República Dominicana, y Uruguay, arrojaron que más de la mitad de los estudiantes de grado sexto de los anteriores países, manifestaron haber sido víctimas de bullying o violencia escolar por parte de uno o varios compañeros de clase. (Unesco, 2005-2009). 
La Facultad de Formación de Profesorado y Educación de la Universidad Autónoma de Madrid (UAM), en el estudio realizado sobre el tema encontró que en América Latina es la región del mundo donde más se presenta la violencia escolar, comparativamente con regiones como África Asia y Europa ese porcentaje oscila entre el 30 y el 40 por ciento".(Roman, 2011, p.34).

\section{Desarrollo Legal y doctrinal}

La Carta Magda de 1991 de Colombia consagró un catálogo de derechos fundamentales, definidos en el ordenamiento internacional como: "los derechos universales e indisponibles establecidos en la Declaración Universal de los Derechos Humanos de 1948, en los pactos internacionales de 1966 y en las demás convenciones internacionales sobre los derechos humanos". (Ferrajoli, 2006), y que fueron asumidos como tales por el Estado Colombiano, en el marco de la dogmática del Estado Social de Derecho.

Estos derechos deben ser respetados por todos los Colombianos, principalmente por las personas que asumen funciones públicas, debido a que ellos son los garantes de los derechos fundamentales, para el caso en comento, es la misma Constitución en el artículo 44 la que establece los derechos de los niños y su prevalencia sobre los derechos de los demás; así como la obligación que tiene la familia, la sociedad y el Estado de asistir y proteger al niño para garantizar su desarrollo armónico e integral y el ejercicio pleno de sus derechos. De otra parte, el artículo 47 dispone que el Estado debe adelantar una política de previsión, rehabilitación e integración social para los disminuidos físicos, sensoriales y psíquicos, a quienes se prestará la atención social que requieran. (Constitución Política, 1991).

Igualmente, la educación en el artículo 67 señala que es un servicio público que cumple una función social, siendo el Estado, la sociedad y la familia responsables de ella, la cual tiene carácter obligatorio entre los cinco y los quince años de edad. Además, determina que el Estado debe garantizar el adecuado cubrimiento del servicio y asegurar las condiciones necesarias para su acceso y permanencia en el sistema educativo, y reconoce a los padres de familia el derecho de escoger el tipo de educación para sus hijos menores.

La educación ha sido reconocida por la Corte Constitucional (Sentencia, T-539) como derecho fundamental por sus características de inherente, inalienable y esencial a la persona humana; igualmente porque contiene principios y normas constitucionales en su consecución y desarrolla que involucran derechos fundamentales de los integrantes de las comunidades educativas, por esta razón ha sido analizada en la Corte Constitucional en sus fallos de revisión desde el año 1992.

Posteriormente, y luego que el Ministerio de Educación Nacional hiciera seguimiento y análisis de la efectividad de esta política en particular; como resultado de su estudio, presentó una iniciativa legislativa al Congreso de la República para contrarrestar la violencia escolar y la comisión de delitos en los entornos educativos; es así como se expide la Ley 1620 de 2013 por la cual se crea el Sistema Nacional de Convivencia Escolar y la Formación para el ejercicio de los Derechos Humanos, la educación para la sexualidad y la prevención y mitigación de la violencia escolar; cuyo objeto es "contribuir a la formación de ciudadanos activos que aporten a la construcción de una sociedad democrática, participativa, pluralista e intercultural.

Es importante destacar que esta ley se promulgó luego que los índices de violencia escolar y embarazos en adolescentes en el país aumentaran considerablemente. La presión mediática también fue trascendental para que el gobierno nacional desde la rama del poder legislativo regulara el tema, creando un sistema de atención integral para los casos de acoso escolar que se presentan en el país. Es así como igualmente 
en la ley se establece la Ruta de Atención Integral para la Convivencia Escolar la cual define los procesos y los protocolos que deberán seguir las entidades e instituciones que conforman el Sistema Nacional de convivencia escolar y formación para los derechos humanos, la educación para la sexualidad y la prevención y mitigación de la violencia escolar, en todos los casos en que se vea afectada la convivencia escolar y los derechos humanos, sexuales y reproductivos de los estudiantes de las instituciones educativas, articulando una oferta de servicio ágil, integral y complementario.

Debido a que la violencia escolar se venía presentado no solamente entre estudiantes sino entre los demás integrantes de las instituciones educativas, llámese directivos, administrativos y docentes; la Ley 115 de 1994 de educación estableció que en todas las Instituciones Educativas debía elaborarse un Manual de Convivencia, herramienta que establece las reglas de conducta que son de obligatorio cumplimiento por toda la comunidad educativa, donde se consagran las políticas escolares, derechos, deberes, conductas sancionables, ruta de atención a las víctimas; siendo autónomas las instituciones en su formulación acorde con los objetivos de la ley de Convivencia escolar. En la elaboración de los Manuales de Convivencia, de acuerdo a la revisión de algunos de ellos, se empezaron a vulnerar derechos fundamentales; según pronunciamientos de la Corte Constitucional, se han presentado situaciones de discriminación en las Instituciones Educativas, como ejemplo señalo el alto tribunal el uso del cabello largo de los estudiantes, la hiperactividad de los niños, el estado de embarazo, las condiciones de sexo, estrato, condiciones físicas y sicológicas; entre otras conductas que han llevado a su pronunciamiento en la mayoría de las ocasiones en defensa de los estudiantes.

\section{Análisis jurisprudencial sobre la Convivencia Escolar en Colombia}

A pesar de contar con herramientas de tipo jurídico, se siguen presentado innumerables problemáticas de difícil solución al interior de las comunidades Educativas relacionadas con las conductas violentas de los integrantes de las mismas; por esta razón se debe acudir en ocasiones al ejercicio de Acciones Constitucionales efectivas como la Acción de Tutela para la protección de Derechos Fundamentales, que en última instancia terminan siendo decididas en acción de revisión por parte de la Corte Constitucional, debido a su trascendencia. Recapitulando; las relaciones entre compañeros, docentes, directivos y estudiantes en su mayoría se construyen en el aula de clase; por esta razón la Corte Constitucional ha resaltado la importancia de la educación en este espacio, debido a que es el lugar semi-privado, en el que se propende la enseñanza, el aprendizaje, el desarrollo personal y social de los alumnos, siendo allí donde se fortalece el desarrollo integral del ser humano. Las aulas de clase, como epicentros de la educación y desde una perspectiva sistémica, han sido definidas como espacios formales e informales que se transforman en centros sociales de participación e interacción entre el estudiante y el docente dentro de ese universo escolar (Quintero Cardozo, 2007, p.4); también han sido caracterizadas como foros de cultura, microsistemas de aprendizaje y enseñanza (Ortega, 1998, p.3) con roles, tensiones y conflictos; espacios de encuentro entre profesores, alumnos y padres de familia; lugares de interacción cultural en donde los procesos de construcción valorativa se constituyen en uno de los fundamentos de todo proceso formativo y son espacios en los que se manifiestan influencias de indole familiar, social y cultural (Guzman, 1991)

La convivencia escolar y la imposición de reglas relacionadas con el espacio, los tiempos y las responsabilidades son factores que unen a los miembros de una comunidad educativa a pesar de sus diferencias; así como los procesos de aprendizaje y enseñanza, promueven el desarrollo de habilidades y, “la integración a la vida social, la participación responsable en la vida ciudadana y en el desarrollo de su propio proyecto de vida". (Berra Bortolotti, 2006)

Justicia Vol. 25 No. 38: pp. 95 - 112. Julio -Diciembre, 2020. https://doi.org/10.17081/just.25.38.4422 
Iniciaremos abordando cronológicamente algunos fallos de la Corte Constitucional relacionados con la convivencia escolar y los derechos fundamentales como el derecho al debido proceso, señalado en los Manuales de Convivencia de las instituciones educativas; derecho que ha sido objeto de protección por parte de la jurisprudencia Constitucional debido a su importancia e inaplicación.

En uno de los casos estudiados, se amparó el derecho de una alumna que había sido encontrada desnuda en una casa ajena, ella interpuso acción de tutela contra la institución educativa argumentando que fue sancionada por una conducta extraña a sus obligaciones, en detrimento de sus derechos al debido proceso, a la intimidad y a la educación. En aquella ocasión, la Corte Constitucional determinó que todas las sanciones que se impongan a los alumnos deben ser públicas, claras y estar previamente establecidas en la ley o reglamento de la institución. Igualmente señaló que "al imponer una sanción se pretende asegurar el cumplimiento de un reglamento interno, sustentado en los principios y valores que orientan el desarrollo de una comunidad académica y que se materializa en la consagración de un conjunto de deberes y prohibiciones, cuya infracción puede ser reprochada por una institución educativa con la condición de que sea respetado el debido proceso" (Corte Constitucional, sentencia T 491, 2003, p.2).

En este fallo se reitera que la función de los docentes en el proceso educativo es de suma importancia, por esta razón, se viene reconociendo jurisprudencialmente la función correctiva de los docentes y directivos de las instituciones educativas, considerando que esa competencia no puede desbordar la verdadera misión del educador contrariando los principios constitucionales y desconociendo los derechos fundamentales de los alumnos.

Como lo establece la Ley general de educación en sus artículos 91 y 92, el educando es el centro del proceso educativo y su formación debe promover el pleno desarrollo de su personalidad, no solamente a través del acceso al conocimiento sino también mediante la educación en valores éticos, morales y ciudadanos que contribuyan al desarrollo socio-económico del país (Ley 115, 1994)

Situaciones como la anterior han sido reiterativas en las Instituciones Educativas, conllevando violaciones a los derechos fundamentales de los estudiantes por parte de los Directivos, e incluso llegando a vulnerarse otros derechos de esta categoría relacionados con la intimidad y el libre desarrollo de la personalidad. Al respecto la Corte ha reiterado que debe establecerse en los Manuales de Convivencia un debido proceso con las garantías constitucionales que conlleva para evitar actuaciones arbitrarias que afecten la convivencia escolar.

Frente al mismo derecho, la corte resolvió el siguiente problema jurídico: ¿violó una institución educativa el derecho al debido proceso, a la educación y a la igualdad de un estudiante, al habérsele adelantado un proceso en el que se le habría impuesto una sanción [matrícula condicional] por haber ingresado a un grupo en una red social que tenía por objeto atacar y difamar a la Rectora del Colegio donde él estudiaba, a pesar de que el Colegio afirma no haber impuesto tal sanción?. Casos como éste han sido analizados por la Corte, corroborando la violación a los derechos fundamentales de los estudiantes (Corte Constitucional, sentencia T 730, 2010, p1).

Para complementar lo señalado, el derecho a la educación debe contemplar la garantía de que el debido proceso como derecho fundamental debe ser aplicado en los trámites disciplinarios en instituciones educativas, en otras sentencias, en casos de similares de hecho y de derecho, la Corte Constitucional ha realizado llamados de atención a las instituciones educativas para que en lo sucesivo no vuelvan a cometer conductas violatorias de este tipo. 
De lo anterior se colige, que, desde la creación de la Corte, la jurisprudencia y a lo largo del tiempo y por más de veinticinco años, se reitera, ha reconocido el carácter fundamental del derecho a la educación; su estrecha relación con el debido proceso a propósito de los trámites que se adelanten en dicho contexto -en especial, si se trata de procesos sancionatorios- y la posibilidad de que la protección del goce efectivo del mismo pueda lograrse mediante la acción de tutela. Son los educadores, quienes orientan el proceso de formación, enseñanza y aprendizaje en las instituciones educativas, cuyo fin es precisamente el de prestar el servicio público de educación con calidad (Corte Constitucional, sentencia T 407, 2012, p.1).

Continuando con el análisis, frente a la violencia escolar; la misma se viene reflejando en la discriminación que se presenta comúnmente en contra de los niños con discapacidad física, mental o auditiva; podría denominarse otra clase de violencia sicológica de los docentes y directivos hacia los menores; al respecto tenemos la sentencia de la Corte Constitucional que señala que es grave para la sicología del estudiante, estigmatizarlo y aislarlo, generando consecuencias no solamente para el pequeño entorno familiar, sino la privación de seres muy valiosos que puedan aportar con su inteligencia y habilidades al desarrollo y al progreso" (Corte Constitucional, sentencia T 255, 2001, p.1).

El mismo fallo otorgó importancia en las consideraciones al tema de la educación inclusiva; para ella "es esencial delimitar el concepto para poder determinar si los medios elegidos como parte de este modelo educativo responden o no a la finalidad del mismo. Una buena manera de entender el concepto de educación inclusiva es el de contrastarlo con otros modelos que han estado presentes en materia de educación de personas con discapacidad. Esta clase de educación-inclusiva-, es la apuesta de diferentes instrumentos internacionales -y que además ha sido acogida por el Ministerio de Educación Nacional4-, busca ampliar el espectro de inclusión de personas con necesidades educativas especiales, más allá del acceso a la escuela regular. La educación inclusiva persigue que no existan ambientes segregados, sino que todos los niños y niñas, independientemente de sus necesidades educativas, puedan estudiar y aprender juntos (Corte Constitucional, sentencia T 255, 2001, p.1) Se analiza la sentencia en cita, debido a que las enfermedades en los niños y adolescentes han aumentado, no solamente de tipo físico sino sicológico; en algunas de las ocasiones utilizadas como excusas por los directivos de planteles educativos para violar derechos fundamentales de los estudiantes en condiciones de debilidad manifiesta, negando su ingreso; su permanencia estudiantil; victimizándolo; dándole un trato diferencial y en otros casos dando un trato discriminatorio a ellos.

Continuando con el análisis; la jurisprudencia ha abordado un tema nuevo relacionado con el aula escolar; hoy llamado: "Proceso restaurativo en los Planteles educativos"; es un trámite autónomo e informado para que la víctima vuelva a restaurar sus derechos y su condición de integrante de la comunidad educativa; igualmente para que tenga claro, según la Corte, se le deben ofrecer al estudiante alternativas, de manera flexible, para no exponerlo a procesos en que no se sienta dignamente tratado; así mismo, la decisión de llevar a cabo este proceso deberá ser expresa, sin perjuicio de que pueda cambiar de parecer. Uno de los problemas identificados que suele presentarse, es que alguno de los disciplinados vuelva a ser o haya seguido siendo parte de la institución educativa. Lo anterior responde a la importancia de verificar la voluntad de seguir para hacer el proceso correspondiente (Corte Constitucional, sentencia 390, 2011, p.1).

Por lo anterior, el trato que se le debe dar a la víctima como al victimario en los procesos que se adelantan en las instituciones educativas, teniendo en cuenta que en la mayoría de ocasiones son personas menores de edad, son de especial interés donde debe existir no solamente el acompañamiento de los padres de familia sino de un equipo interdisciplinar cuando el caso no pueda ser manejado por el comité de convivencia escolar.

4 Ministerio de Educación Nacional. 
De otra parte, debe precisarse que los pronunciamientos del alto tribunal sobre la temática abordada, han generado reflexiones al interior del Ministerio de Educación Nacional de Colombia; incluso en algunos de sus fallos se ha ordenado vincularlo al igual que a otras entidades públicas y privadas como el Instituto Colombiano de Bienestar Familiar, la Procuraduría General de la Nación, Defensoría del Pueblo y Universidades; dada la importancia y las funciones que cada una de ellas cumple en la Educación en Colombia.

Un claro ejemplo de lo mencionado, se materializa en la Sentencia de la Corte Constitucional T 905 de 2011, donde se ordenó vincular a la Universidad del Norte para escuchar los argumentos de la academia sobre el tema; conceptuando este ente de educación superior a través de la Dirección de Sicología lo siguiente: "las prácticas de matoneo pueden ser entendidas como una situación de acoso, intimidación o victimización en la que el alumno es atacado por un compañero o grupo de compañeros. Se trata de actos repetitivos, prolongados en el tiempo y además, se evidencia un desbalance de poder entre víctima y agresor" (Corte Constitucional, sentencia T 905, 2011, p.1).

En la misma providencia se diferenciaron cinco prácticas a través de las cuales se manifiesta este género de conducta (físico, verbal, exclusión social, mixto y cyberbullying), advirtiendo la Corte que sólo de manera reciente la sociedad colombiana ha dirigido su atención sobre el fenómeno, debido "posiblemente a la aparición de casos con consecuencias nefastas tanto para agresores como víctimas". (Corte Constitucional, Sentencia t 905, 2011).

Como consideraciones de la Corte en la misma sentencia, se referenció la investigación realizada por Uninorte, frente a los niños y niñas de la Costa Caribe, señalando: “En el 2009 se llevó a cabo el último estudio de incidencia financiado por el Observatorio de educación de la Universidad del Norte. Este estudio tuvo una muestra de 1642 estudiantes entre 6o y 110 grado de colegios de (sic) públicos del departamento del Atlántico. Los resultados del estudio revelan que $88,7 \%$ de los niños afirman haber presenciado situaciones de agresión, $19,7 \%$ se reconocen como agresores y $40,7 \%$ como víctimas. Además, los abusos más frecuentes entre compañeros son las agresiones verbales -hablar mal (30,5\%), poner apodos (46,3\% e insultar (25,7\%)- seguidas de las agresiones físicas indirectas y directas -esconder las cosas $(21,1 \%$ ) y pegar (13,5\%)" (Corte Constitucional, sentencia T 905, 2011, p.11).

Lo anterior fue un indicador negativo que mostró el aumento de los niveles de violencia escolar, situación que debía poner en contexto al Ministerio de Educación para que asumiera el ejercicio de sus funciones y creara políticas para contrarrestar esta problemática.

Vale la pena mencionar, cómo el Ministerio de Educación Nacional Colombiano, a través de la Jefe de la Oficina Asesora Jurídica se ha pronunciado frente a casos concretos analizados por la Corte; reiterando el régimen de competencias definido en las Leyes 115 de 1994 y 715 de 2001; con base en ello reiteró que su papel es sólo el de fijar las políticas generales en materia de educación y "no administra, o contrata los servicios educativos", ni tampoco es el superior jerárquico de las Secretarías de Educación; afirmación que permite inferir que se está fallando en los procesos de administración del sistema, tal como se señala por parte del Ministerio, las políticas existen, simplemente no se han efectivizado en cada plantel educativo: ¿de quién será la responsabilidad en este sentido?

Siguiendo con el análisis de la sentencia, en su parte resolutiva se determinó entre otros aspectos: "Ordenar Ministerio de Educación Nacional que en el término de seis meses a partir del fallo, en coordinación con el Instituto Colombiano de Bienestar Familiar, la Defensoría del Pueblo y la Procuraduría General de la Nación, lidere la formulación de una política general que permita la prevención, la detección y la atención de las prácticas de hostigamiento, acoso o "matoneo escolar", de manera que sea coherente con los pro- 
gramas que se adelantan en la actualidad, con las competencias de las entidades territoriales y que constituya una herramienta básica para la actualización de todos los manuales de convivencia. La aplicación de tales instrumentos deberá tener como objetivo inmediato a las directivas, profesores, estudiantes y padres de familia; conforme a lo dispuesto en este numeral, el establecimiento educativo tendrá un plazo no mayor a nueve meses para la modificación de su manual de convivencia, término durante el cual deberá definir y poner en marcha una estrategia provisional para reconocer la vulneración de derechos fundamentales en este caso y para implementar ejercicios de tolerancia y respeto" (Corte Constitucional, sentencia T 904, 2011, p.21)

Analizando lo señalado, la Corte continúa sentando precedentes jurisprudenciales sobre el tema y persiste en hacer llamados de atención en sus providencias; para que de manera perentoria los organismos correspondientes formulen las políticas tendientes a menguar este problema.

De acuerdo a lo anterior, se hace la reflexión en el sentido de que ha sido la Corte Constitucional la más informada de la problemática que nos ocupa y la más interesada en que se lograra efectivizar la función del Estado; nótese como fueron un sinnúmero de casos relacionados con la convivencia escolar que llegaron al alto tribunal, precisamente por el desconocimiento de la Constitución Política de 1991 por parte de las Instituciones Educativas al elaborar los Manuales de Convivencia y aplicar incorrectamente y con desconocimiento la Ley y el debido proceso.

Es por esto, y debido al cúmulo de casos fallados por el alto tribunal que se le ha dado especial protección a los menores víctimas de abusos y a las instituciones educativas; se observa como en sus sentencias se viene protegiendo la identidad de las partes (accionante y accionada); siendo común que se les cambie los nombres, siendo remplazados por letras; tal como se señala a continuación: "Por su parte, se instará al Colegio AA, para que desarrolle una política escolar que permita la prevención, oportuna detección, atención y protección, frente al hostigamiento, acoso o matoneo escolar, incluyendo el llamado "ciber matoneo" o "cyberbullying", con el fin de evitar que situaciones similares a las que se presentan en este caso vuelvan a suceder en detrimento de los derechos fundamentales de los estudiantes". (Corte Constitucional, Sentencia T 365, 2015).

En Colombia, en el año 2014 se presentó un caso que conmovió a todo el país; el estudiante Sergio Urrego de 17 años quien cursaba grado once en un Colegio privado de Bogotá fue objeto de bullying por parte de la Comunidad educativa; donde se le adelantó un proceso disciplinario por descubrirse su condición sexual a través de unas fotos de celular; situación que lo llevó a tomar la decisión de suicidarse el día 4 de agosto del mismo año, arrojándose de la terraza de un Centro Comercial en dicha ciudad; su madre al ver vulnerados los derechos fundamentales de su hijo ya fallecido, decidió interponer una acción de tutela con los siguientes argumentos:

Alba Lucía Reyes Arenas, presentó acción de tutela el 11 de septiembre de 2014 a nombre propio y en representación de su difunto hijo Sergio David Urrego Reyes, en contra del colegio Gimnasio Castillo Campestre, la Secretaría de Educación de Cundinamarca, el Instituto Colombiano de Bienestar Familiar, la Fiscalía General de la Nación y la Comisaría Décima de Familia de Engativá, por considerar, en primer lugar, que las directivas de la institución educativa demandada, promovieron conductas sistemáticas de discriminación en contra su hijo, motivadas por su orientación sexual, -tanto en el proceso disciplinario que se surtió en su contra, como con la información que fue difundida con posterioridad al fallecimiento del niño en los medios de comunicación-, que favorecieron inicialmente su suicidio y que resultaron finalmente lesivas de sus derechos fundamentales. En segundo lugar, frente a las demás entidades estatales acusadas en la tutela, estima que éstas desplegaron una conducta omisiva ante las diferentes denuncias que se realizaron con 
fundamento en las actuaciones equivocadas de la institución educativa, por lo que incurrieron a su vez, en la violación de los derechos fundamentales del menor de edad (Corte Constitucional, Sentencia 478, 2015).

Situación que luego de la expedición de la Ley de Convivencia Escolar, no tendría por qué seguirse presentando en Colombia, con el agravante que las conductas violatorias de la Institución Educativa y otras entidades como el Ministerio de Educación acarrearon el suicidio de un menor de edad.

En este sentido, la Corte Constitucional profirió el fallo, precedente judicial para futuras configuraciones de violación de derechos en las comunidades educativas y los correspondientes llamados de atención, primeramente concedió la protección de los derechos fundamentales a la intimidad y buen nombre, igualdad y no discriminación, libre desarrollo de la personalidad, el derecho a la educación, la prevalencia de los derechos de los menores de edad y el derecho al debido proceso de la accionante al joven SERGIO URREO (qepd) ya fallecido por las actuaciones de acoso escolar y discriminación de las que fueron objeto por parte del Colegio Gimnasio Castilla Campestre; de la misma manera se ordenó al representante legal del Colegio Gimnasio Castillo Campestre que, en un plazo máximo de quince (15) días contados a partir de la notificación de la presente sentencia, realizara en las instalaciones de la institución, con la presencia activa de la comunidad educativa, el Ministerio de Educación Nacional, los representantes de los grupos y colectivos a los que perteneció Sergio, sus familiares y amigos, un ACTO PÚBLICO DE DESAGRAVIO en donde se reconozcan las virtudes de Sergio y su legado y se reconozca el respeto que se le debía brindar a su proyecto de vida. (Corte Constitucional, Sentencia 478, 2015).

En el mismo, se deberán realizar los siguientes actos: i) conceder el grado póstumo al menor; ii) instalar y develar una placa en memoria del joven con la siguiente inscripción: "una educación ética es el único mecanismo para obtener la perfección, destino último de los ciudadanos. La misma solo es posible si enseñamos en la diferencia, la pluralidad y el imperativo absoluto de respetar a los demás". En memoria de Sergio David Urrego Reyes. 1997-2014"; iii) una declaración pública por parte de las autoridades del Colegio Gimnasio Castillo Campestre donde se reconozca que la orientación sexual que asumió Sergio debía ser plenamente respetada en el ámbito educativo y que los foros educativos son espacios de tolerancia y respeto encaminados a construir un ciudadano respetuoso de las diversas posturas y comprometido con la igualdad en la diferencia; y iv) una declaración del Gobierno Nacional, en cabeza del Ministerio de Educación Nacional, donde se comprometa de manera pública a desarrollar acciones, en el marco de los módulos del Programa para la Educación Sexual y Construcción de Ciudadanía, para promover el respeto por la diversidad sexual en los colegios" (Corte Constitucional, sentencia T 478, 2015, p.9).

Respecto a la efectividad de la Ley 1620 de 2013, en coherencia con el citado fallo, se resalta lo resuelto por la Corte Constitucional en el sentido que reitera y vuelve a ordena al Ministerio de Educación Nacional que, en un plazo máximo de seis (6) meses contados a partir de la notificación de la sentencia, implemente acciones tendientes a la creación definitiva del Sistema Nacional de Convivencia Escolar de acuerdo a lo señalado por la Ley 1620 de 2013 y el Decreto 1965 de 2015. Particularmente, se ordena que en el plazo señalado, se adopten las siguientes medidas: i) conformar, si no lo ha hecho aún, el Comité Nacional de Convivencia Escolar y verificar, en el mismo plazo, que todos los comités municipales, distritales y departamentales de convivencia escolar estén funcionando plenamente; ii) implementar, si no lo ha hecho aún, al programa para el desarrollo de competencias ciudadanas, la educación para el ejercicio de los derechos humanos -en particular el derecho a la identidad sexual- e incorporarlos de manera expresa en los proyectos educativos institucionales de todos los colegios del país; iii) desarrollar y poner en práctica el Sistema de Información Unificado de Convivencia Escolar, garantizando el respeto por la intimidad y confidencialidad de las personas involucradas; y iv) establecer la Ruta de Atención Integral para la Convivencia Escolar, junto a sus Protocolos y en un plazo máximo de un (1) año contado a partir de la notificación de la presente 
sentencia, implemente acciones tendientes a la creación definitiva del Sistema Nacional de Convivencia Escolar de acuerdo a lo señalado por la Ley 1620 de 2013 y el Decreto 1965 de 2015. Particularmente, se ordena que en el plazo señalado, se adopten las siguientes medidas: i) una revisión extensiva e integral de todos los Manuales de Convivencia en el país para determinar que los mismos sean respetuosos de la orientación sexual y la identidad de género de los estudiantes y para que incorporen nuevas formas y alternativas para incentivar y fortalecer la convivencia escolar y el ejercicio de los derechos humanos, sexuales y reproductivos de los estudiantes, que permitan aprender del error, respetar la diversidad y dirimir los conflictos de manera pacífica, asi como que contribuyan a dar posibles soluciones a situaciones y conductas internas que atenten contra el ejercicio de sus derechos; y ii) ordenar y verificar que en todos los establecimientos de educación preescolar, básica y media estén constituidos los comités escolares de convivencia" (Corte Constitucional, sentencia T 478, 2015, p.11)

La misma Corte Constitucional quiso en este fallo a través de la Sala Administrativa del Consejo Superior de la Judicatura, difundir por el medio más expedito posible la sentencia a todos los despachos judiciales del país, para que fuera aplicada en casos de acoso escolar, institucional o particular, las medidas preventivas necesarias de acuerdo al Sistema Nacional de Convivencia Escolar y la obligación de proteger el derecho a la educación de los menores, así como garantizar que el mismo sea un espacio de respeto por la diversidad, la pluralidad y la igualdad en la diferencia. Con lo anterior se concluye que, pasados dos años de expedición de la Ley y su decreto reglamentario, a través de un fallo de orden constitucional se llamó nuevamente la atención a las entidades respectivas para que asumieran sus funciones e implementaran la Ley de convivencia escolar en los planteles educativos. Hoy, aún existen dificultades en la implementación de la Ley en los Manuales de Convivencia, su reglamentación y el funcionamiento de los comités de convivencia escolar.

Para finalizar el análisis, el día 23 de marzo del año 2017, el Tribunal Administrativo de Boyacá, en fallo proferido por la Magistrada Ponente Clara Elisa Cifuentes en Medio de Control Reparación Directa, expediente 15001-3333-009-2014-00135-01, fue declarado responsable el Municipio de Tunja de las lesiones sufridas al menor Rafael ocurridas el día 31 de mayo de 2012 en el Instituto Técnico Gonzálo Suarez Rendón y ordenó una condena por perjuicios morales, materiales y de daño a la salud derivados de los hechos que se resumen a continuación:

Rafael, estudiante del Colegio Gonzalo Suarez Rendón, quien cursaba grado sexto en la jornada de la mañana, el día 31 de mayo de 2012 siendo las 10 de la mañana fue objeto de agresión por parte de otro estudiante, impactando su ojo derecho y dando como resultado la pérdida total del órgano lesionado.

El fallo en mención, igualmente hizo un enérgico llamado de atención al Municipio de Tunja- Secretaria de Educación Municipal y le ordenó: "que en el término de un mes elabore y apruebe los documentos internos pertinentes para la adopción de políticas tendientes a crear conciencia sobre la necesidad de garantizar la integridad física de los estudiantes, velar por el comportamiento de los estudiantes dado que las instituciones Educativas responden por las conductas antijurídicas de estos en tanto están bajo la tutela del Colegio o la Institución Educativa respectiva y la adopción de medidas para que la infraestructura se encuentre en condiciones que no constituya riesgo para la salud de los estudiantes, como sucedió en el sub lite (Fallo Medio de Control Reparación Directa en contra del Municipio de Tunja, 2017). 


\section{A manera de conclusiones}

En este artículo, se puede concluir que las sentencias de la Corte Constitucional y el Tribunal Administrativo de Boyacá, obedecen a faltas originadas en la ausencia de capacitación, desconocimiento e inaplicación de la Ley de convivencia escolar y seguimiento por parte del Ministerio de Educación Nacional Colombiano a las Instituciones Educativas en todo el País. Si bien éste solamente formula las políticas respectivas igualmente debe velar por la implementación y efectividad de las mismas, a pesar de la autonomía que tienen las instituciones educativas de crear su Manual de Convivencia.

Como lo ha manifestado la Corte en la sentencia T-124 de 1998, la "autorregulación de los centros de educación no es absoluta, sino que debe estar enmarcada en el respeto de los derechos y fines constitucionales y legales y que debe proveer procedimientos claros y expresos en los Manuales de Convivencia, definido por los mismos miembros de la comunidad educativa".

Han sido reiterados los llamados de atención, especialmente por la Corte Constitucional al Ministerio de Educación Nacional para que implemente y desarrolle planes y programas tendientes al mayor cubrimiento de una buena formación especializada de los docentes del país, con el fin de que puedan asumir en debida forma la tarea de educar a los niños y jóvenes; evitando que sigan recibiendo maltrato, estigmatización y aislamiento social, productos de su condición especial diferente.

Igualmente, se estableció que esta política pública de convivencia escolar que surgió y se concretó en una Ley, como consecuencia de un estado de necesidad latente en las Instituciones Educativas; que requería una normatividad que sustentara jurídicamente sus decisiones; no ha logrado cumplir a cabalidad los objetivos para los cuales fue creada.

La apuesta pasa por una revisión crítica y análisis jurisprudencial; estableciendo que existe una inoperancia de la Ley de Convivencia, concluyendo que no ha sido suficiente y efectiva su implementación en las Instituciones Educativas para el adecuado manejo de los conflictos.

Finalmente se puede afirmar que ha sido la Corte Constitucional la que ha realizado el seguimiento a la implementación de la Ley de Convivencia escolar en las Instituciones Educativas siendo de responsabilidad del Ministerio de Educación Nacional Colombiano su control y no de este máximo Tribunal Constitucional.

\section{Referencias}

Abramovay, M. (2005). Victimización en las escuelas. Revista Mexicana de Investigación Educativa, 833-864. Bandura, A. (2010). El Aprendizaje Social y el Desarrollo de la Personalidad.

Berger, C. \&. (2009). Violencia escolar. Estudios y posibilidades de intervención en Latinoamérica. Santiado de Chile: Universitaria.

Berra Bortolotti, D. F. (2006). Convivencia Escolar y Habilidades Sociales. Revista Científica Electrónica de Psicología.

Blaya, C., Debarbieux, É., \& Rey-Alamillo, R. d.-R. (2005). Clima y violencia escolar. Un estudio comparativo entre España y Francia. Revista de Educación., 339-293-31.

Carolina \& Koller, S. (2009). Factores protectores y de riesgo para la agresividad y victimización en escolares brasileños: el rol de los amigos. Violencia, la violencia en las escuelas., 415-428.

Castillo Pulido, L. E. (2011). El acoso escolar. De las causas, origen y manifestaciones a la pregunta por el sentido que le otorgan los actores. Revista internacional de investigación en Educación, 415-430. 
Chaux, E. (2002). Buscando pistas para prevenir la violencia urbana en Colombia:onflictos y agresión entre niños y adolescentes de Bogotá. Bogotá: Universidad de los Andes.

Chaux, E. (2003). Agresión reactiva, apresión instrumental y el ciclo de la violencia. Revista Estudios Sociales, 47-58.

Constitución Política (Congreso de la República 1991).

Corte Constitucional (2011). Sentencia T 390 de 2011. MP. Joge Iván Palacio Palacio.

Corte Constitucional (2015). Sentencia T 478 de 2011. MP. Gloria Stella Ortiz Delgado.

Corte Constitucional (2014).Sentencia T 804 de 2014. MP. Jorge Iván Palacio Palacio.

Corte Constitucional (2014).Sentencia T 365 de 2014. MP. Nelson Pinilla Pinilla.

Corte Constitucional (2012). Sentencia T 407 de 2014. MP. Mauricio González Cuervo.

Corte Constitucional (2003). Sentencia T 491 de 2003. MP. Clara Inés Vargas Hernández.

Corte Constitucional (2010) Sentencia T 730 de 2003. MP. Mauricio González Cuervo.

Corte Constitucional (2011). Sentencia T 905 de 2011. MP. Jorse Iván Palacio Palacio.

Corte Constitucional (2001). Sentencia T-255 de 2011. MP. Jose Gregorio Hernández.

Domenach, 1. (1981).

Fallo Medio de Control Reparación Directa en contra del Municipio de Tunja, 2014-135-01 (Contencioso Administrativo de Boyacá 23 de Marzo de 2017).

Ferrajoli, L. (2006). Sobre los derechos fundamentales. Cuestiones Constitucionales, 113-136.

Flores González, L. (2009). Interpretaciones fenomenológicas sobre el sentido de la violencia escolar. Christian Berger \& Carolina Lisboa (eds.)., 211-230.

Guzman, B. G. (1991). El aula: espacio de interrelación de quehaceres y finalidades educativas. El aula universitaria, UNAM, México.

Hoyos, O., Aparicio, J., \& Heilbron, K. \&. (2004). Representación sobre el maltrato entre iguales en niñas y niños escolarizados de 9, 11 y 13 años de nivel socioeconómico alto y bajo en la ciudad de Barranquilla, Colombia. Sicología desde el Caribe, 150-172.

Instituto para la Investigación Educativa y el Desarrollo Pedagógico, I. (1999). Violencia en la escuela. Bogotá: Alcaldía Mayor de Santafé de Bogotá.

Ley 115 (1994). Ley General de Educación.

Ley 1620 (2013). Ley de Convivencia Escolar.

Medina Gallego, C. (1991). Escuela y violencia: una reflexión desde la cotidianidad escolar. Educación y Cultura, 24.

Olweus, D. (1978). Aggression in the schools: Bullies and whipping boys. Washington D.C., USA.

Olweus, D. (2004). Conductas de acoso y amenaza entre escolares.

OMS. (2003). Informe mundial sobre la violencia y la salud. Washington: Scientific and Technical Publications.

Ortega. (1998). La convivencia escolar: Que es y cómo abordarla. Consejería de Educación y Ciencia.

Ortega-Ruiz, R. (2002). Lo mejor y lo peor de las redes de iguales: juego, conflicto y violencia. Revista Interuniversitaria de Formación de Profesorado, 93-113.

Quintero Corzo, J. M. (2007). Aula Investigativa: un espacio para construir saber pedagógico. Investigación-Acción y Currículo; Un recorrido por el Mundo., 123-142. Obtenido de http://bibliotecadigital. conevyt.org.mx/servicios/hemeroteca/reencuentro/no26/Aula/Aula.htm. 
Rodríguez, V. (2011). El manual de convivencia escolar y el libre desarrollo de la personalidad. Una visión jurisprudencial. Justicia Juris., 17-26.

Román, M. M. (2011). América Latina : Violencia entre estudiantes y desempeño escolar. Revista Cepal.

Tamara-Yunes. (1993). Mortalidad por causas violentas en adolescentes y jóvenes: un desafío para la región de las Américas. Revista Brasilera de Epidemiología, 102-171.

Unesco. (2005-2009). Informe Violencia Escolar.

Unicef. (2011). Violencia escolar en Ámerica Latina y el Caribe. Superficie y fondo. Panamá.

Zimbardo, P. (2012). El efecto Lucifer. El Por qué de la Maldad. 
